\title{
Absolute Scale Quantitative Off-Axis Electron Holography at Atomic Resolution
}

\author{
Florian Winkler, ${ }^{1,2}$ Juri Barthel, ${ }^{1,3}$ Amir H. Tavabi, ${ }^{1,2}$ Sven Borghardt, ${ }^{4}$ \\ Beata E. Kardynal, ${ }^{4}$ and Rafal E. Dunin-Borkowski ${ }^{1,2}$ \\ ${ }^{1}$ Ernst Ruska-Centre for Microscopy and Spectroscopy with Electrons (ER-C), Forschungszentrum Jülich, 52425 Jülich, Germany \\ ${ }^{2}$ Peter Grünberg Institute 5 (PGI-5), Forschungszentrum Jülich, 52425 Jülich, Germany \\ ${ }^{3}$ Gemeinschaftslabor für Elektronenmikroskopie (GFE), RWTH Aachen University, 52074 Aachen, Germany \\ ${ }^{4}$ Peter Grünberg Institute 9 (PGI-9), Forschungszentrum Jülich, 52425 Jülich, Germany
}

(Received 30 January 2018; published 11 April 2018)

\begin{abstract}
An absolute scale match between experiment and simulation in atomic-resolution off-axis electron holography is demonstrated, with unknown experimental parameters determined directly from the recorded electron wave function using an automated numerical algorithm. We show that the local thickness and tilt of a pristine thin $\mathrm{WSe}_{2}$ flake can be measured uniquely, whereas some electron optical aberrations cannot be determined unambiguously for a periodic object. The ability to determine local specimen and imaging parameters directly from electron wave functions is of great importance for quantitative studies of electrostatic potentials in nanoscale materials, in particular when performing in situ experiments and considering that aberrations change over time.
\end{abstract}

DOI: 10.1103/PhysRevLett.120.156101

Transmission electron microscopy (TEM) is a powerful and versatile technique for imaging the microstructure of materials at atomic resolution. Structural details that may have a significant influence on material properties, such as atomic arrangements, displacements, and local variations in composition at defects, interfaces, and surfaces can be identified by the quantitative analysis of TEM images [1]. A frequently applied approach to quantitative TEM is to verify a structure model by matching experimental images to simulations, ideally on the same absolute scale, by using a forward modeling approach. In recent years, significant advances have been made by achieving absolute scale matches between experimental images and simulations in both conventional coherent high-resolution TEM and incoherent scanning TEM [2-5]. Important prerequisites that have enabled these developments include careful calibration of the detector sensitivity and accurate characterization of the electron microscope [6-8].

Despite the availability of aberration correctors and highly stable electron microscopes, residual aberrations, partial coherence effects, vibrations, external fields, and detector properties can all have a significant influence on the recorded signal [9-12]. As a result, a large number of instrumental parameters need to be included in image simulations, in addition to atomic structure models. Although many instrumental parameters can in principle be determined independently by performing additional experiments [13-16], electron optical aberrations can vary rapidly over time [17], meaning that the optical state of the instrument during an experiment is often only partially known. Other parameters, such as the local sample thickness, tilt, drift, and vibrations, are either unpredictable or very difficult to measure with sufficient accuracy.

Some of these difficulties can, in principle, be removed if the full complex-valued electron wave function is recorded using a technique such as off-axis electron holography. The phase of the object-modulated wave function is highly sensitive to important material properties, such as local variations in charge density, dopant potential, and magnetization [18-24]. The application of electron holography in a modern electron microscope that has a spatial resolution of below $0.1 \mathrm{~nm}$ should therefore provide access to both the structure and the functional properties of materials with atomic spatial resolution at the same time. However, the ability to use simulations that match experimentally recorded wave functions quantitatively at atomic spatial resolution is required for reliable interpretation of the recorded signal, in order to realize the full potential of electron holography.

Here, we demonstrate an absolute scale match of simulated and experimental electron wave functions recorded using off-axis electron holography at atomic resolution. We implement a forward modeling approach in the form of a fully automated optimization algorithm, which is used to determine unknown experimental parameters from a recorded wave function of a pristine thin $\mathrm{WSe}_{2}$ flake, whose atomic structure is assumed to be known. In this way, previous attempts to determine experimental parameters from wave functions [25-27] are surpassed on a quantitative level by replacing generic symmetry arguments with matches to realistic simulations. The regions of known crystal structure that are required for our approach can often be found directly next to areas of 
interest, e.g., in support materials or pristine volumes surrounding a defect $[2,3]$.

For the automated determination of a large set of unknown parameters that describe both the imaging conditions of the electron microscope and the specimen, it is advantageous if subsets of the parameters can be determined independently, thereby reducing the dimensions of the search spaces, while increasing the number of optimization problems to be solved. Here, we separate the parameters into two sets: (A) Parameters that essentially affect the amplitudes of the Fourier coefficients, and (B) parameters that only affect the phases of the Fourier coefficients.

Parameter set (A) contains six, usually unknown parameters to be determined: the local specimen tilt is described by a two-dimensional vector $\left(t_{x}, t_{y}\right)$; absorption is modeled by calculating an absorptive potential as a fraction $\kappa$ of the elastic scattering potential [28]; an anisotropic Gaussian envelope is applied to the Fourier coefficients, in order to simulate an image spread with major axis $\sigma_{1}$, minor axis $\sigma_{2}$, and orientation $\alpha$ with respect to the horizontal image axis, describing the effects of thermal magnetic field noise, mechanical vibrations, and electrical instabilities $[29,30]$. Although the specimen thickness can also be included as a parameter during optimization, in layered materials such as that investigated here, the thickness can be measured independently by analyzing the mean phase and amplitude, as described in our previous work [31].

The determination of parameter set (A) requires the calculation of electron diffraction within the specimen, e.g., by the multislice method [32]. Since this calculation cannot be described in a closed analytical form, the gradient of the cost function to be minimized can only be calculated numerically. In order to minimize the computational demand, a non-gradient-based optimization algorithm, such as the Simplex method described by Nelder and Mead [33,34], can be used. Agreement between simulation and experiment is quantified by the cost function

$$
\begin{aligned}
\Delta^{2}= & \sum_{g}\left|A^{\exp }(g)-A^{\operatorname{sim}}(g)\right|^{2} \\
& +2 A^{\exp }(0) A^{\operatorname{sim}}(0)\left[1-\cos \left(\phi^{\exp }(0)-\phi^{\operatorname{sim}}(0)\right)\right],
\end{aligned}
$$

where the first term accumulates the squared differences of the Fourier coefficient amplitudes $A^{\exp }(g)$ and $A^{\operatorname{sim}}(g)$ for diffraction vectors $g$, and the second term describes the difference between the mean phase values $\phi^{\exp }(0)$ and $\phi^{\mathrm{sim}}(0)$ weighted by the product of the mean amplitudes.

The mean phase of the wave function measured using off-axis electron holography is sensitive not only to the mean electrostatic potential in the specimen, but also to small mistilts of the sample from a crystallographic zone axis [31]. It is therefore highly beneficial to include the mean phase in the cost function. For this purpose, potentials derived from density functional theory (DFT) provide more accurate estimates of the mean inner potential than those obtained using independent atomic form factors [19]. We follow this approach here by using electrostatic potentials for $\mathrm{WSe}_{2}$ calculated using the DFT-based method described in the same work.

The simulated wave function determined using parameter set (A) can be regarded as the wave function at the exit plane of the sample. Comparison with the experimental wave function then allows the determination of parameter set (B), which comprises the coherent aberrations of the imaging system. The effects of aberrations can be removed from the experimental image wave function $\Psi_{\mathrm{i}}(g)$ by applying an exponential phase factor $\exp [i \chi(g)]$, thereby obtaining an experimental exit-plane wave function

$$
\Psi(g)=\Psi_{\mathrm{i}}(g) \exp [i \chi(g)] .
$$

The aberration function $\chi(g)$ is a polynomial series written in terms of the diffraction vector, containing all of the coherent aberration terms that are relevant for a specific instrument. Since $\chi(g)$ and its derivative can be calculated analytically, gradient-based optimization algorithms that typically converge faster than non-gradient-based algorithms can be used. Here, a Sequential Least Squares Programming (SLSQP) algorithm [34,35] was applied to minimize the total squared difference

$$
\Delta_{\mathrm{rms}}^{2}=\sum_{g}\left|\Psi^{\operatorname{Exp}}(g)-\Psi^{\operatorname{Sim}}(g)\right|^{2},
$$

where $\Psi^{\operatorname{Exp}}(g)$ and $\Psi^{\operatorname{Sim}}(g)$ are the complex-valued Fourier coefficients of the experimental and simulated exit-plane wave functions, respectively. In this way, strong influences of Fourier coefficients with low signal-to-noise ratios on the parameter estimation are avoided.

Here, aberrations up to second order were included as variables in the minimization, which comprised in total nine coefficients: image shift $A_{0}$, defocus $C_{1}$, twofold astigmatism $A_{1}$, coma $B_{2}$, and threefold astigmatism $A_{2}$ [36]. Additional knowledge about strong higher-order aberration coefficients was obtained from independent measurements performed using the Zemlin tableau method [13]. The measurements indicate the presence of spherical aberrations with $C_{S}=16 \mu \mathrm{m}$ and $C_{5}=-6.5 \mathrm{~mm}$, which are both considered to be constant.

In the present study, we applied the forward modeling approach to analyze electron wave functions of ultrathin $\mathrm{WSe}_{2}$ with the $2 \mathrm{H}$ crystal structure recorded using off-axis electron holography. $\mathrm{WSe}_{2}$ forms pristine surfaces due to the absence of surface reconstructions and belongs to the class of layered transition metal dichalcogenides that have thickness variations in integer multiples of a single layer [37], simplifying the determination of sample thickness, as large areas of constant thickness can often be found.

Figure 1 shows the phase of a reconstructed and normalized electron wave function of $\mathrm{WSe}_{2}$, with a vacuum 


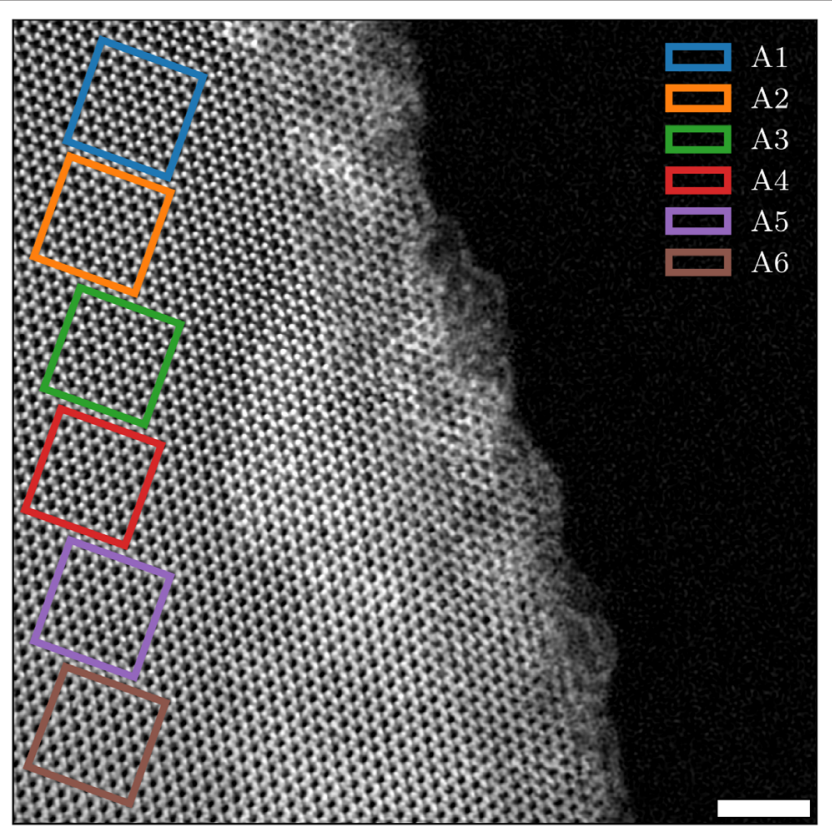

FIG. 1. Electron optical phase image of a $\mathrm{WSe}_{2}$ flake. The wave function was reconstructed from an off-axis electron hologram, with a $40 \mathrm{mrad}$ aperture applied to the sideband. Colored rectangles mark six subregions A1-A6 of $7 \times 4$ orthorhombic unit cells selected for further analysis. The scale bar is $2 \mathrm{~nm}$. The gray scale covers phase values from $0 \mathrm{rad}$ (black) to $1.2 \mathrm{rad}$ (white).

region on the right and a clean area on the left. The bright nonuniform contrast close to the specimen edge is associated with surface contamination. The specimen thickness was measured to be precisely five layers $(3.9 \mathrm{~nm})$ by analyzing the mean phase and amplitude [31].

The electron holograms were recorded using the $C_{S}$ and $C_{C}$ corrected PICO microscope [38] operated at $80 \mathrm{kV}$ with identical experimental conditions as described in Ref. [31]. After acquisition, the detector modulation transfer function (MTF) was deconvolved from both object and reference holograms. Reconstruction of the holograms was carried out using a Python-based algorithm [39] by applying a $40 \mathrm{mrad}$ aperture with a smoothness of $1 \mathrm{mrad}$ to a sideband in Fourier space. Normalization was achieved by (i) registration against a reference electron hologram recorded with the same instrument settings from a vacuum region [40] and (ii) subsequent removal of a residual phase ramp extrapolated from the vacuum region close to the specimen edge $[41,42]$

Six nonoverlapping regions across the image from the top (A1) to the bottom (A6) were selected for detailed analysis, each containing $7 \times 4$ orthorhombic unit cells. Commensurate sampling with the periodic structure yields sharp peaks in the Fourier transform of the wave function, as shown in Fig. 2. The diffraction pattern corresponds to the hexagonal crystal structure visible in the phase image in all of the selected sample areas. However, the maxima in the phase image do not exhibit the circular shapes that are

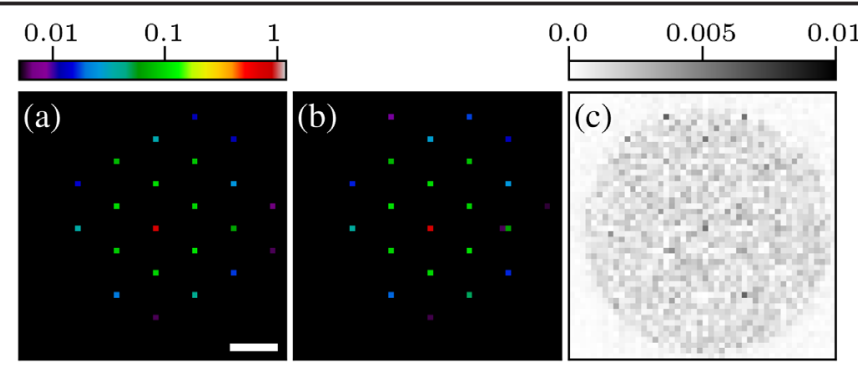

FIG. 2. Comparison of experimental and simulated Fourier coefficients. Amplitudes of the Fourier coefficients of (a) the experimental and (b) the best matching simulated wave function in region A1 plotted on a logarithmic color scale. (c) Absolute differences between experimental and simulated amplitudes plotted on a linear gray scale. The scale bar in the diffraction patterns corresponds to $4 \mathrm{~nm}^{-1}$.

expected for projections of atomic columns, as a result of a combination of residual aberrations, sample mistilt, drift, and anisotropic vibrations.

Electron wave functions were simulated using a multislice approach, as implemented in the Dr. Probe software package $[32,43]$. Electron scattering potentials were derived from electrostatic potentials of $\mathrm{WSe}_{2}$ calculated using DFT, as described in Ref. [19]. In addition, a global Debye-Waller factor with $B=0.003 \mathrm{~nm}^{2}$ [44] was applied to the potentials to take into account the damping effect of thermal atom vibrations. A comprehensive list of all parameters known from independent measurements and unknown parameters determined by automated forward modeling is given in Table S1 in the Supplemental Material [45].

The results of the automated procedure used to determine the parameters for set (A) in region A1 are shown in the form of diffraction patterns in Fig. 2 (see Fig. S1 in the Supplemental Material for all regions [45]). The quality of the match is confirmed from the residual amplitude differences, which are, on average, 2 orders of magnitude smaller than the experimental amplitudes. Values of the best-fitting parameters for all six regions are plotted in Fig. 3.

An anisotropic image spread was consistently found for all of the regions, with $\sigma_{1}=38.3 \pm 0.6 \mathrm{pm}$ and $\sigma_{2}=27.8 \pm 0.3 \mathrm{pm}$. The anisotropy and the larger value when compared to the estimate of $25 \mathrm{pm}$ made in previous work [30] are most likely a result of the longer exposure times used for off-axis electron holography. The absorption parameter $\kappa$ shows no systematic change across the field of view. The fitted value of $7.5 \% \pm 0.5 \%$ is in the range obtained by others $[2,3,46]$. In contrast, a systematic variation in specimen tilt is observed across the image, indicating a bending of the $\mathrm{WSe}_{2}$ flake. Remarkably small errors of less than $0.1^{\circ}$ are obtained for the measured values of specimen tilt. To the best of our knowledge, this is one of the most precise local specimen orientation measurements achieved so far using TEM for very thin samples [47-49]. 


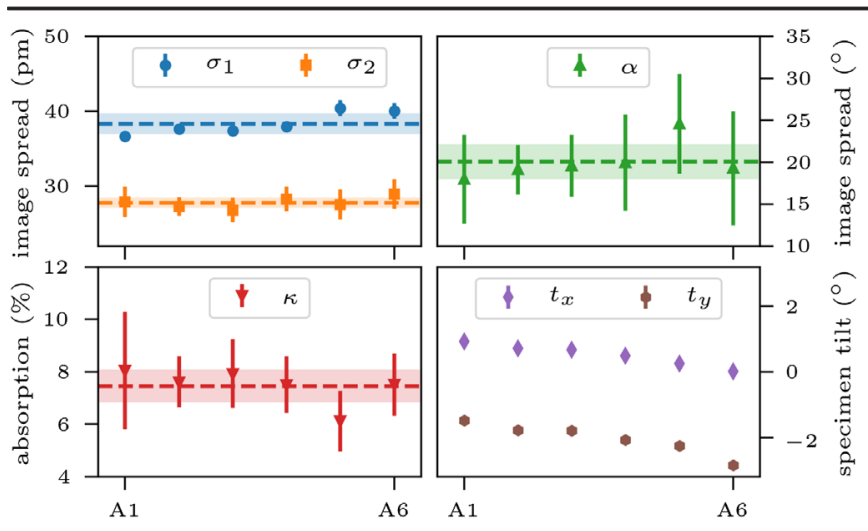

FIG. 3. Fitted experimental parameters. The upper two plots show the image spread parameters $\sigma_{1}, \sigma_{2}$ (left) and $\alpha$ (right), with standard deviations indicated by confidence bands around the mean value (dashed line). The lower plots show the absorption parameter $\kappa$ (left) and local specimen tilts $t_{x}$ and $t_{y}$ (right). The error bars are estimated by means of the covariance matrix calculated at the minimum of the optimization using a linear approximation of the Jacobian matrix.

The uniqueness of the solution was confirmed by repeated optimization trials started at random points in the six-dimensional search space. The reliability and robustness of the algorithm was tested successfully in a statistical approach, in which experimental data were substituted by simulations that included a realistic amount of recording noise for a large set of different parameter scenarios. We found that, for similar noise amplitudes as in the experimental data, the algorithm always converged to the correct solution with uncertainties in the determined parameter values that were even smaller than those obtained in the experimental case.

The strong effects of residual aberrations on the details of the atomic structure in the wave function are visualized in Fig. 4 for area A1 (cf. Figs. S2 and S3 in the Supplemental

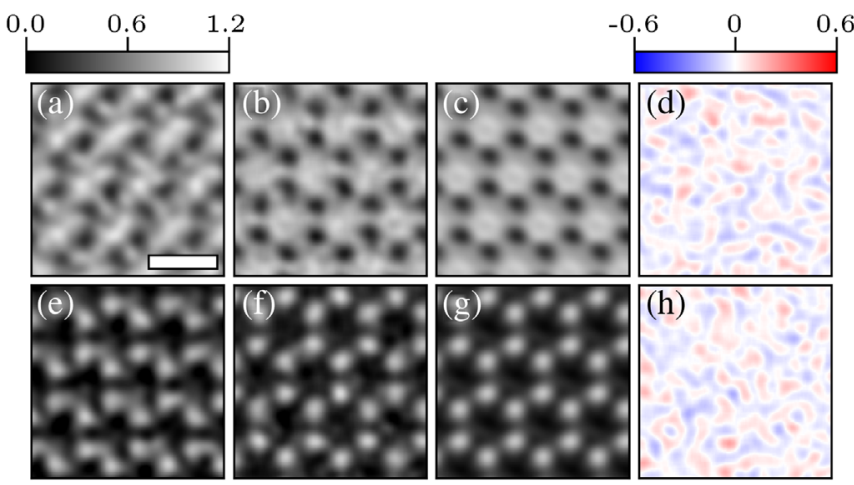

FIG. 4. Comparison between experimental and simulated wave functions. (a)-(d) Real parts and (e)-(h) imaginary parts of the electron wave functions corresponding to region A1. Experimental wave functions (a),(e) before and (b),(f) after residual aberration correction. (c),(g) Simulated wave function. (d),(h) Residual differences between simulation and experiment after aberration correction. The scale bar is $0.4 \mathrm{~nm}$.
Material for results from all of the regions [45]). After aberration correction, the experimental and simulated wave functions match remarkably well. The residual differences displayed on the right show no prominent features that are related to the crystal structure. In all six regions, the rootmean-square deviation $\Delta_{\mathrm{rms}}$ is between 0.076 and 0.090 , which is very close to the vacuum noise level of the experimental wave function (0.072).

Equally good correspondence between simulation and experiment was found for several different aberration sets by starting the optimization at random points in the search space. The existence of multiple equally low minima for $\Delta_{\mathrm{rms}}$ is a consequence of the periodic nature of the exponential phase factor $\exp [i \chi(g)]$. In combination with a sparse and regular excitation of Fourier coefficients, aberration functions that differ exactly by multiples of $2 \pi$ at the positions of the excited beams result in identical values for $\Psi(g)$ in Eq. (2) (cf. Fig. S4 in the Supplemental Material [45]).

In our experiments, we expect that temporal changes between prior aberration correction and the acquisition of the hologram are below $5 \mathrm{~nm}$ for $C_{1}$ and $A_{1}$ and below $1 \mu \mathrm{m}$ for $A_{2}$ and $B_{2}$. We found that $A_{2}$ is particularly difficult to determine, as its rotational symmetry corresponds well to the hexagonal symmetry of the $\mathrm{WSe}_{2}$ [001] diffraction pattern. The equivalent solutions that are found here are separated by at least $50-100 \mathrm{~nm}$ in both $A_{2}$ and $B_{2}$, which can usually be distinguished by the Zemlin tableau method. Table S2 and Fig. S5 in the Supplemental Material show the results of at least four possible solutions found in region A1 [45].

In all of the possible aberration sets that were found here, essentially all of the aberration coefficients show a linear variation across the field of view (cf. Table S3 and Fig. S6 in the Supplemental Material [45]), with the exception of the defocus change, which is related to the local sample tilt of the $\mathrm{WSe}_{2}$ flake. A spatial variation of the lower-order aberrations is induced by the present $C_{\mathrm{S}}$ and $C_{5}$ aberrations, in combination with a large variation of the incident beam tilt over the field of view. The beam tilt is caused by the elliptical illumination used for off-axis electron holography, with a strong variation expected along the direction of the smallest beam diameter [50]. The local object tilt determined in the previous step therefore reflects a combination of both specimen tilt and illumination tilt.

The automated numerical algorithms described here converged fast, with minimal human bias. In particular, the convergence times were about 2 min for the determination of parameter set (A) and $30 \mathrm{~s}$ for the determination of parameter set (B) for each area using a single CPU on a standard desktop computer. This enabled us to analyze the solutions on a statistical basis, showing that parameters which essentially affect the amplitudes of the Fourier coefficients can be determined accurately and unambiguously. In contrast, we found multiple solutions, which 
provide equally good agreement between experiment and simulation, for the residual coherent aberrations. This ambiguity occurs, in general, for periodic objects and is often overlooked or ignored in the process of numerical aberration estimation and correction. The measurement of aberrations shortly before or after the acquisition of electron holograms is probably the most feasible way to identify the correct solution, or at least to limit the number of possible aberration sets. Another possibility to resolve the ambiguity is to analyze other crystalline structures, or the same structure at a different zone axis orientation, if this is available in the same wave function.

The automation of the forward modeling approach realized in our work allowed us to investigate multiple smaller areas of the recorded wave function in a reasonable time. In the present case, we found that many experimental parameters vary dramatically over the field of view. Beamtilt-induced variations of lower-order aberrations may be a general phenomenon in electron holography experiments performed using elliptical illumination. Therefore, separating the field of view into multiple smaller areas may be a suitable approach for the quantitative analysis of electron holograms.

The primary requirement for the presented procedure is a knowledge of the investigated crystal structure. In practice, known crystal structures can often be found next to a region of interest. For example, structural defects are often embedded in pristine volumes of material. By dividing the image into subregions, the unknown experimental parameters can be measured in pristine areas and extrapolated to the region of interest. Another possibility would be to use support materials of known structure, such as graphene. In such cases, the procedure can be applied to determine the experimental parameters from regions that contain the known structure, in order to simplify subsequent analysis of an unknown structural feature in close proximity.

In summary, we have demonstrated absolute scale agreement between experimental and simulated electron wave functions, which is limited only by the experimental recording noise and potential sample contamination. This agreement has been achieved by using an innovative numerical procedure, which exploits all of the information carried by the complex-valued wave function in an electron hologram. A particularly important aspect of the procedure involves automation, which allows statistical analysis of the robustness of the solution and the analysis of large areas. By analyzing large areas that are subdivided into multiple smaller regions, we demonstrated the locality of the obtained results, finding a clear bending of the investigated sample. Significant spatial variations of some of the experimental parameters, such as the coherent aberrations, were found. This variation is particularly relevant for the quantification of localized defects, for which inaccurate aberration correction could lead to incorrect conclusions. With regard to the limited lifetime of optical states for atomic resolution TEM, the determination of imaging parameters directly from experimental images may be the only practical way to perform fully quantitative analysis during longer time-resolved experiments.

We are grateful to J. Caron, J. Ungermann, M. Lentzen, and M. Linck for valuable discussions and to Z. Zanolli and M. Verstraete for support with DFT calculations. The research leading to these results has received funding from the European Research Council under the European Union's Seventh Framework Programme (No. FP7/20072013)/ ERC Grant Agreement No. 320832. We are grateful for computing time granted by the John von Neumann Institute for Computing (NIC) and provided on the supercomputer JURECA in the Jülich Supercomputing Centre (JSC) (JARA-HPC Projects No. JIAS16 and No. JPGI90).

* Corresponding author.

f.winkler@fz-juelich.de

[1] K. W. Urban, Science 321, 506 (2008).

[2] C. L. Jia, S. B. Mi, J. Barthel, D. W. Wang, R. E. Dunin-Borkowski, and K. W. Urban, Nat. Mater. 13, 1044 (2014).

[3] C. L. Jia, J. Barthel, F. Gunkel, R. Dittmann, S. HoffmannEifert, L. Houben, M. Lentzen, and A. Thust, Microsc. Microanal. 19, 310 (2013).

[4] B. D. Esser, A. J. Hauser, R. E. A. Williams, L. J. Allen, P. M. Woodward, F. Y. Yang, and D. W. McComb, Phys. Rev. Lett. 117, 176101 (2016).

[5] A. Rosenauer, K. Gries, K. Müller, A. Pretorius, M. Schowalter, A. Avramescu, K. Engl, and S. Lutgen, Ultramicroscopy 109, 1171 (2009).

[6] A. Thust, Phys. Rev. Lett. 102, 220801 (2009).

[7] J. M. LeBeau, S. D. Findlay, L. J. Allen, and S. Stemmer, Nano Lett. 10, 4405 (2010).

[8] C. Dwyer, C. Maunders, C. L. Zheng, M. Weyland, P. C. Tiemeijer, and J. Etheridge, Appl. Phys. Lett. 100, 191915 (2012).

[9] M. Haider, H. Rose, S. Uhlemann, E. Schwan, B. Kabius, and K. W. Urban, Ultramicroscopy 75, 53 (1998).

[10] O. L. Krivanek, N. Dellby, and A. R. Lupini, Ultramicroscopy 78, 1 (1999).

[11] K. Ishizuka, Ultramicroscopy 5, 55 (1980).

[12] J.C.H. Spence, Experimental High-Resolution Electron Microscopy (Oxford University Press, New York, 1988).

[13] F. Zemlin, K. Weiss, P. Schiske, W. Kunath, and K. H. Herrmann, Ultramicroscopy 3, 49 (1978).

[14] R. R. Meyer, A. I. Kirkland, R. E. Dunin-Borkowski, and J. L. Hutchison, Ultramicroscopy 85, 9 (2000).

[15] J. Barthel and A. Thust, Phys. Rev. Lett. 101, 200801 (2008).

[16] C. Dwyer, R. Erni, and J. Etheridge, Appl. Phys. Lett. 93, 021115 (2008).

[17] J. Barthel and A. Thust, Ultramicroscopy 134, 6 (2013).

[18] P. A. Midgley and R. E. Dunin-Borkowski, Nat. Mater. 8, 271 (2009). 
[19] S. Borghardt, F. Winkler, Z. Zanolli, M. J. Verstraete, J. Barthel, A. H. Tavabi, R. E. Dunin-Borkowski, and B. E. Kardynal, Phys. Rev. Lett. 118, 086101 (2017).

[20] H. Lichte, P. Formanek, A. Lenk, M. Linck, C. Matzeck, M. Lehmann, and P. Simon, Annu. Rev. Mater. Res. 37, 539 (2007).

[21] D. Cooper, C. Ailliot, J. P. Barnes, J. M. Hartmann, P. Salles, G. Benassayag, and R. E. Dunin-Borkowski, Ultramicroscopy 110, 383 (2010).

[22] J. Li, M. R. McCartney, and D. J. Smith, Ultramicroscopy 94, 149 (2003).

[23] Z. Li, F. Zheng, A. H. Tavabi, J. Caron, C. Jin, H. Du, A. Kovács, M. Tian, M. Farle, and R. E. Dunin-Borkowski, Nano Lett. 17, 1395 (2017).

[24] H. Lichte, M. Reibold, K. Brand, and M. Lehmann, Ultramicroscopy 93, 199 (2002).

[25] C. Ophus, H. I. Rasool, M. Linck, A. Zettl, and J. Ciston, Adv. Struct. Chem. Imaging 2, 15 (2017).

[26] D. Tang, H. W. Zandbergen, J. Jansen, M. Op de Beeck, and D. Van Dyck, Ultramicroscopy 64, 265 (1996).

[27] D. Stenkamp, J. Microsc. 190, 194 (1998).

[28] H. Hashimoto, A. Howie, and M. J. Whelan, Proc. R. Soc. A 269, 80 (1962).

[29] S. Uhlemann, H. Müller, P. Hartel, J. Zach, and M. Haider, Phys. Rev. Lett. 111, 046101 (2013).

[30] M. Haider, P. Hartel, H. Müller, S. Uhlemann, and J. Zach, Microsc. Microanal. 16, 393 (2010).

[31] F. Winkler, A. H. Tavabi, J. Barthel, M. Duchamp, E. Yucelen, S. Borghardt, B. E. Kardynal, and R. E. Dunin-Borkowski, Ultramicroscopy 178, 38 (2017).

[32] J. M. Cowley and A. F. Moodie, Acta Crystallogr. 10, 609 (1957).

[33] J. A Nelder and R. Mead, Computer Journal (UK) 7, 308 (1965).
[34] E. Jones et al., http://www.scipy.org/.

[35] D. Kraft, Technical Report No. DFVLR-FB 88-28, Institut für Dynamik der Flugsysteme, Deutsche Forschungs- und Versuchsanstalt für Luft- und Raumfahrt (DFVLR), 1988.

[36] D. Typke and D. Köstler, Ultramicroscopy 2, 285 (1977).

[37] K. A. N. Duerloo, Y. Li, and E. J. Reed, Nat. Commun. 5, 4214 (2014).

[38] J. Barthel, L. Houben, and K. Tillmann, J. Large-Scale Res. Facil. 1, A34 (2015).

[39] F. De la Peña et al., http://doi.org/10.5281/zenodo.345099, 2017.

[40] M. Linck, B. Freitag, S. Kujawa, M. Lehmann, and T. Niermann, Ultramicroscopy 116, 13 (2012).

[41] E. Völkl, L.F. Allard, and D. C. Joy, Introduction to Electron Holography (Springer, New York, 1999).

[42] H. Lichte, D. Geiger, A. Harscher, E. Heindl, M. Lehmann, D. Malamidis, A. Orchowski, and W. D. Rau, Ultramicroscopy 64, 67 (1996).

[43] J. Barthel, http://www.er-c.org/barthel/drprobe/, 2017.

[44] F. Z. Chien, Y. S. Chen, Y. C. Chu, W. Y. You, J. B. Lee, T. E. Dann, C. H. Tu, and Y. S. Huang, Chin. J. Phys. 26, 119 (1988).

[45] See Supplemental Material at http://link.aps.org/ supplemental/10.1103/PhysRevLett.120.156101 for aberration coefficients and visualization of the corresponding aberration functions of at least four possible solutions determined in region A1.

[46] L. Jin, J. Barthel, C. L. Jia, and K. W. Urban, Ultramicroscopy 176, 99 (2017).

[47] H. H. Liu, S. Schmidt, H. F. Poulsen, A. Godfrey, Z. Q. Liu, J. A. Sharon, and X. Huang, Science 332, 833 (2011).

[48] Q. Liu, Ultramicroscopy 60, 81 (1995).

[49] X. Huang and Q. Liu, Ultramicroscopy 74, 123 (1998).

[50] M. Lehmann, Ultramicroscopy 100, 9 (2004). 